\title{
The effect of the heat treatment level HT on the mechanical behavior of cork
}

\author{
T. Kermezli*, M. Announ**, A. Bensmaili**** \\ *Department of Material Engineering, University of Medea, Algeria, E-mail: t_kermezli@yahoo.fr \\ **Laboratory of Materials and environment, LME, University of Medea, Algeria, E-mail: moh_announ@yahoo.fr \\ ***LGR, University of Science and Technology H Boumedien, Algeria, E-mail: abensmaili@yahoo.fr \\ cross $^{\text {ref }}$ http://dx.doi.org/10.5755/j01.mech.22.1.12252
}

\section{Introduction}

The requirement of thermal and acoustic comfort in the field of civil engineering, it is very important to assess the durability of mechanical and acoustic behaviour of building biomaterials, through qualitative and quantitative analyses of chemical species flow in the pores of the biomaterials. For the cork industry, the analysis of physico- chemical changes provides important information on the thermal and sound barrier as well as the drying energy consumption [1-4].

The exposure of cork to high temperatures causes significant changes in its chemical structure [5]. The magnitude of the change depends on the temperature level and duration of exposure to heat which leads to permanent reduction in the strength of the intermolecular links. This reduction is probably due to the depolymerization reactions. The failure of chemical bonds starts at a temperature above $100^{\circ} \mathrm{C}[6,7]$.

In order to know the effect of biodegradation on one hand and to allow for the optimization of the consumption of agents during the treatment process on another hand, it is therefore very important to know the routine over time the diffusing chemical species into the material at different levels of temperature. Many studies have been performed on the mass transfer in various anisotropic materials. They are presented in the literature, but especially for the natural cork and its derivatives very few studies of the properties concerning their electrical and dielectric properties are found in scientific literature $[5,8]$.

To this purpose, experiments were carried out in order to understand in particular the diffusion coefficient of material, where the effect of the cycle of the processing temperature is quantified by monitoring the desorption kinetics of the tracer $(\mathrm{NaCl})$ through the voids of the anisotropic medium "cork" in the radial direction.

In the first step, the kinetics is governed by a mathematical model where the mass equation is solved by adopting the appropriate simplifying assumptions as well as appropriate boundary conditions (material and experimental protocol). In the second step the diffusion coefficient is assessed at each cycle of treatment temperature of the material.

By doing so, this will give us the ability to determine the treatment cycle which is appropriate for the best performances of cork.

First cross-linking in the lignin is demonstrated by a hardening of the material. Then, and according to the same authors [4], the change in the crystallography of cellulose decreases the material bending strength.
Degradation of Hemicellulose and cellulose decreases the water uptake, resulting in increase in durability of the material against the attacks of micro-organisms. The modelling of moisture transfer in cork requires the accurate knowledge of several thermophysical and boundary condition parameters that may appear in the formulation.

In literature, the models describing migration in porous media of water usually in vapour form. The aim of this paper is to present analysis of mass transfer in liquid form before and after heat treatment of Algerian cork.

This work is based on the indirect method of conductimetric measurement for following the kinetics of desorption of the aqueous solution $(\mathrm{NaCl}$ " $0.2 \mathrm{M}$ "). This method has been revealed very simple in its material conception, highly accurate and the results thereof immediately obtainable. The aqueous solution diffuses radially and longitudinally within an anisotropic porous medium (Cork in form of plate before and after treatment) towards an external environment.

As determination of the diffusivity by conventional methods requires long and laborious steps, the conductivity method constitutes infact, a good alternative which requires less effort. In this context, the apparent diffusion coefficient Dap of cork has been determined successive assessment starting from a mathematical model.

Indeed, by analysing an impregnated porous material in a aqueous solution, the model key entry is the reduced mass from the experimentally measured conductivity. The experimental database is used to develop a methodology that will be employed to determine the diffusion coefficient by a statistical approach based on Artificial Neural Networks, which will be a future study.

\section{Experimental protocol}

The experimental protocol consists of a preparation of cork chips from Akfadou (Algeria). The geometric properties are shown in Table 1, whereas the spatial orientation of the pores is illustrated in Fig. 1.

Table 1

Cork chips Dimensions used in the experimental protocol (radial direction)

\begin{tabular}{|l|c|c|}
\hline & Side squar, $\mathrm{m}$ & Thickness 21, $\mathrm{m}$ \\
\hline Plate C1 & $34.510^{-3}$ & $4.1010^{-3}$ \\
\hline Plate C2 & $34.010^{-3}$ & $4.0410^{-3}$ \\
\hline Plate C3 & $34.010^{-3}$ & $4.1810^{-3}$ \\
\hline
\end{tabular}




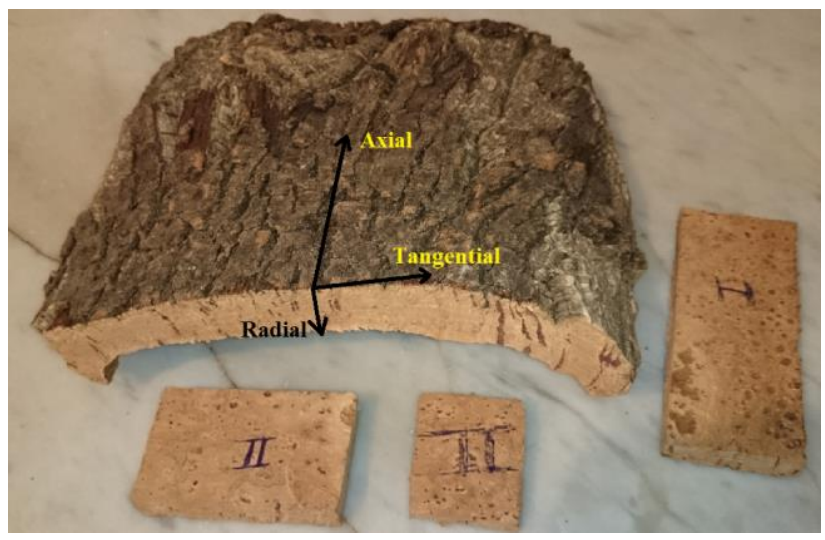

Fig. 1 Illustration of the three main directions of the sample in a section of cork

Biomaterial was analyzed by thermogravimetrically method. This method allows to measure the mass loss [9] and to get an idea of the range of degradation temperature of the cork components (hemicellulose, cellulose, lignin) as well.

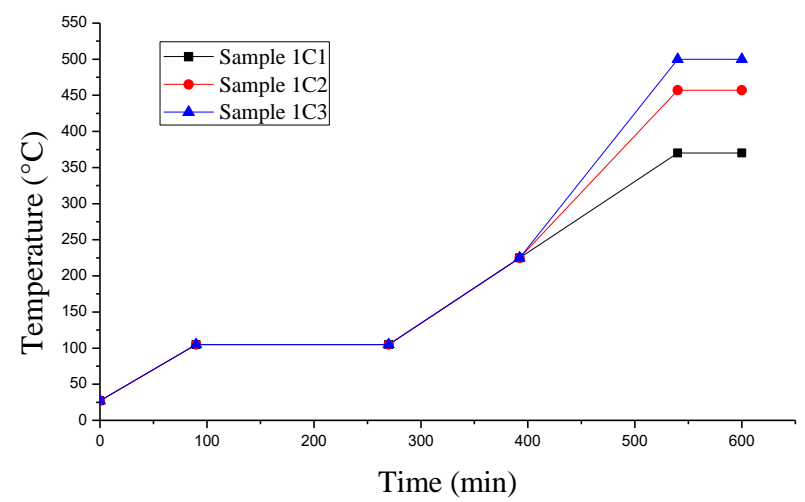

a

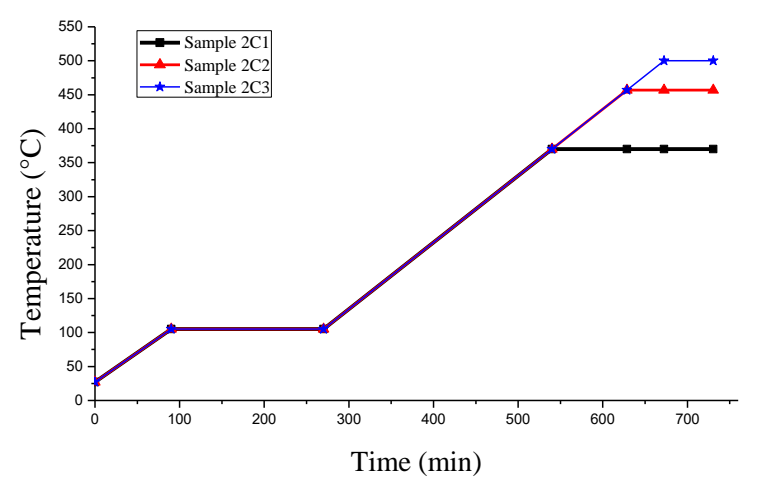

b

Fig. 2 Heat treatment cycles a - constant step; b - variable step

Fig. 2 shows the samples subjected to heat treatment at various temperature cycle and different treatment steps.

In the experimental protocol (a) the rate of treatment was maintained at $1{ }^{\circ} \mathrm{C} / \mathrm{min}$. Whereas, in the case of the experimental protocol (b) beyond the $225^{\circ} \mathrm{C}$ the rate was changed for each level, respectively $1{ }^{\circ} \mathrm{C} / \mathrm{min}$ for cork chips $1 \mathrm{C} 1^{\left(370^{\circ} \mathrm{C}\right)}, 1.5^{\circ} \mathrm{C} / \mathrm{min}$ for $1 \mathrm{C} 2^{\left(457^{\circ} \mathrm{C}\right)}$ and $2^{\circ} \mathrm{C} / \mathrm{min}$ for $1 \mathrm{C} 3^{\left(500^{\circ} \mathrm{C}\right)}$.

The choice of temperature cycles is justified by the onset of thermal degradation of the cork which starts at about $225^{\circ} \mathrm{C}$. The hemicellulose components begin to decompose at about $225^{\circ} \mathrm{C}$ and most of them degrade at $325^{\circ} \mathrm{C}$. The cellulose polymer is more stable towards the thermal degradation as it begins to decompose at a temperature of $370^{\circ} \mathrm{C}$, while the lignin degrades progressively over.

These chips are subjected to impregnation by a solute $(0.2 \mathrm{M} \mathrm{NaCl})$ for good traceability and availability for 24 hours to a whole saturation (Fig. 3).

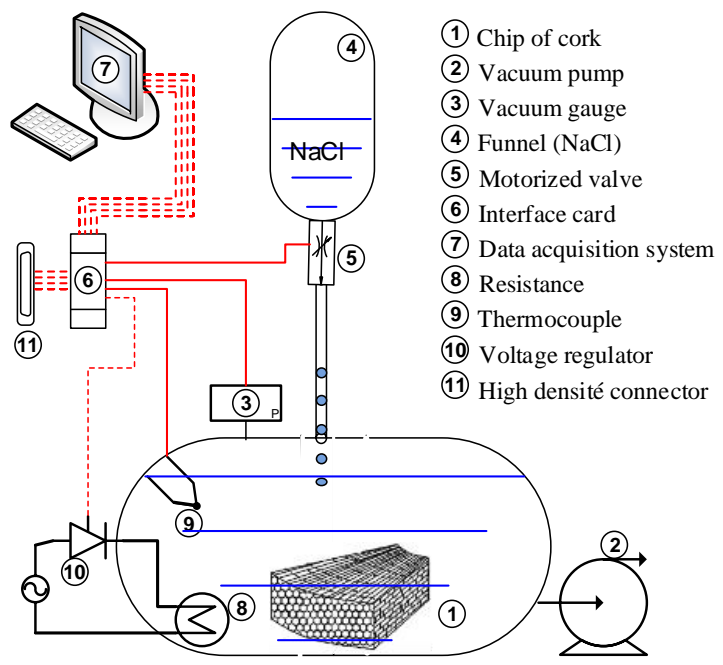

Fig. 3 Schematic drawing of vacuum impregnation of the cork sample

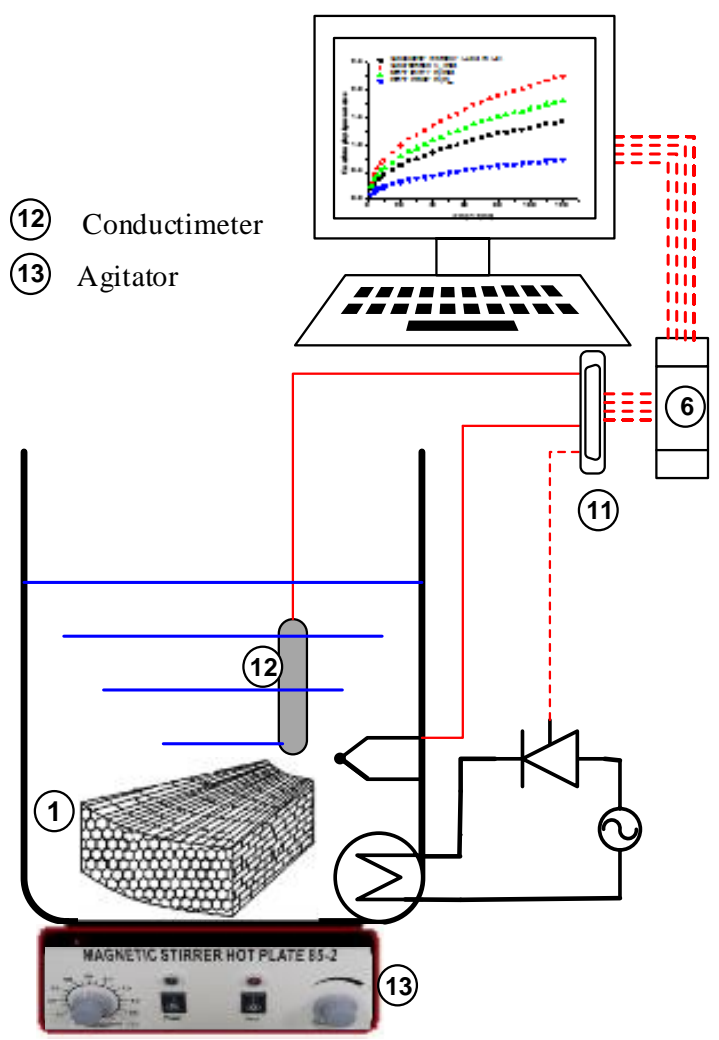

Fig. 4 Schematic drawing of the experimental measurement of the concentration of desorption

The mass of $\mathrm{NaCl}$ was weighed to prepare solutions using the following equation:

$$
m_{\mathrm{NCl}}=c_{\mathrm{NaCl}} m_{\mathrm{NaCl}} V_{\mathrm{H}_{2} \mathrm{O}}=29.222 \mathrm{~g} \text {. }
$$


The solute concentration of the desorption is calculated indirectly according to initial and instant conductivity indicated by the conductimeter and the cationic and anionic equivalent conductivity at the temperature $T$ :
Eqs. 2 and 3 [10].

$$
c_{\mathrm{NaCl}}=\frac{\sum_{i}\left(\lambda_{i} z_{i} c_{i}\right)}{\lambda_{\mathrm{Na}^{+}}(T)+\lambda_{\mathrm{Cl}^{-}}(T)}
$$

Table 2 equivalent conductivity limits diffusion of the cation Values of the coefficients (Eq. 3) and/or anion in water at $25^{\circ} \mathrm{C}$.

\section{Modeling}

The procedure to develop a mathematical model based on mass balance with the objective of determining the diffusion coefficient Diap during the desorption process, using the concentration distribution inside the composite which has a finite and simple geometry. where $\lambda_{i}$ the ion equivalent conductivity " $i$ "; $c_{i}$ the ions concentration $\left(\right.$ gr. $\left.{ }^{-1}\right) ; T$ the solution temperature $(\mathrm{K})$; $\Lambda_{\mathrm{NaCl}}$ the conductivity measured by the conductimeter; $\lambda_{x}^{0}$

$$
\frac{\partial c_{i}\left(X_{i}, F_{0 j}\right)}{\partial F_{0 j}}-D_{i a p}\left[\frac{\partial^{2} c_{i}\left(X_{j}, F_{0 j}\right)}{\partial X_{j}^{2}}-\frac{\beta}{X_{i}} \frac{\partial c_{i}\left(X_{i}, F_{0 j}\right)}{\partial F_{j}}\right] \pm \frac{\xi^{2} R_{i}}{D_{i a p}\left(c_{i p}-c_{i 0}\right)}+\frac{\xi}{D_{i}} \frac{\partial\left[c_{i}\left(X_{j}, F_{0 j}\right) U_{c}\right]}{\partial \xi}=0 .
$$

After simplification, we obtain:

$$
\cdot \frac{\partial c_{i}\left(X_{j}, F_{0 j}\right)}{\partial F_{0 j}}-D_{i a p}\left[\frac{\partial^{2} c_{i}\left(X_{j}, F_{0 j}\right)}{\partial X_{j}^{2}}-\frac{\beta}{X_{j}} \frac{\partial c_{i}\left(X_{j}, F_{0 j}\right)}{\partial X_{j}}\right]=0 .
$$

Our model is based on the mass balance of the diffusing chemical species for different geometries of the

$$
c_{R i}=1-\frac{4}{\pi} \sum_{n=0}^{\infty}\left\{\frac{(-1)^{n}}{2 n+1} \cos \left[\frac{2 n+1}{2 l} \pi x\right] \exp \left[-\left(\frac{2 n \pi+\pi}{2 l}\right)^{2} D_{\text {iap }} t\right]\right\},
$$

where $c_{i}$ is the diffusing concentration, $D_{i a p}$ is the apparent diffusion coefficient, $c_{R i}$ is the reduced concentration of the chemical species " $i$ " and 21 is the thickness. The diffusion coefficient was determined from the Eq. 6 using iterative calculation which gives the best fit of the reduced mass released experimental data as is shown in Fig. 5.

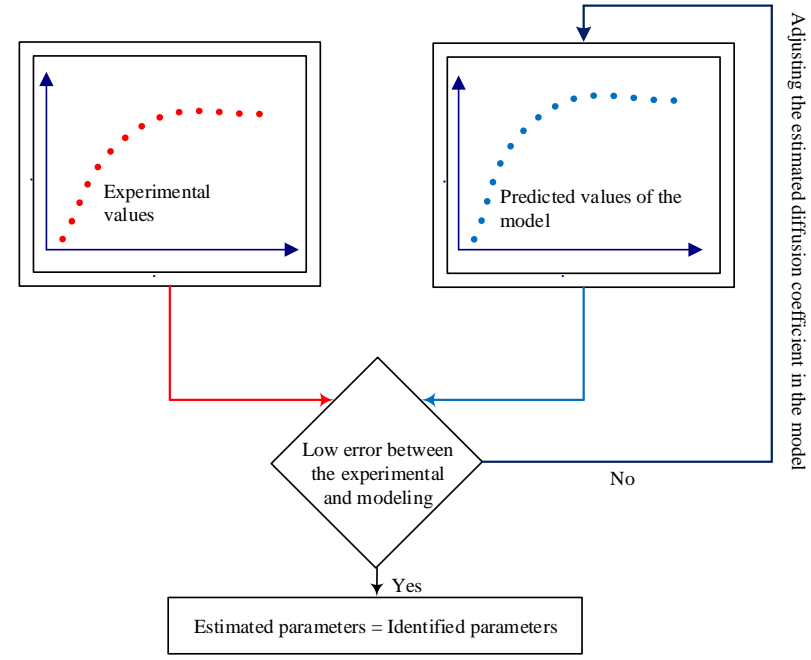

Fig. 5 Computation algorithm of the diffusion coefficient

\section{Results and Interpretations}

Fig. 6 shows the TGA curve of the mass loss of $2 \mathrm{C}$ cork, which allows us to visualize and illustrates the different levels of chemical elements degradation that causes their biodegradation. Several phases were distinguished based on the level of temperature:

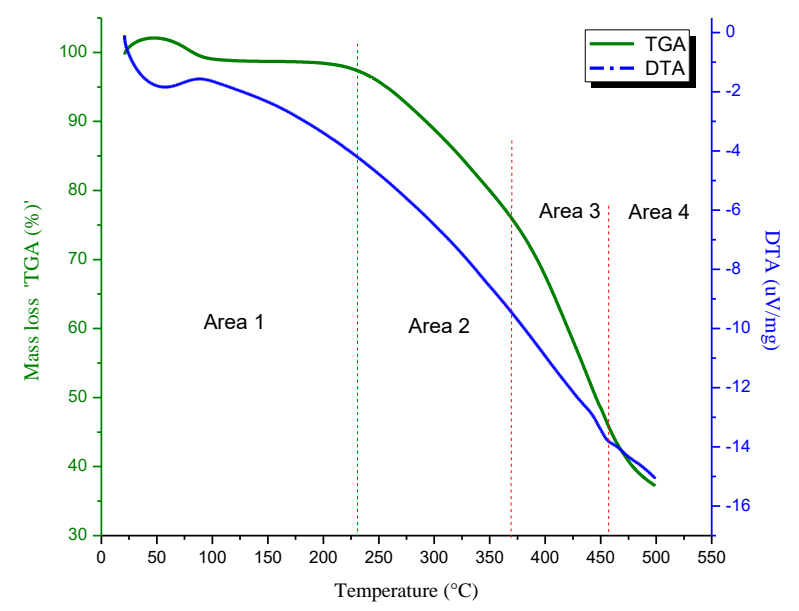

Fig. 6 TGA analysis of cork 
The first area $\left(T<200^{\circ} \mathrm{C}\right)$ corresponding to the drying phase during that the residual moisture is removed. The second zone between $230^{\circ} \mathrm{C}$ and $370^{\circ} \mathrm{C}$ is characterized by the loss of hemicellulose degradation. The change of slope of the curve occurs at the beginning of the third area which is between $370^{\circ} \mathrm{C}$ and $457^{\circ} \mathrm{C}$; this trend indicates that there is a chemical change in kinetics of cellulose degradation. The fourth zone above $457^{\circ} \mathrm{C}$ is where the degradation of lignin occurs. This graph shows clearly that the kinetic of lignin degradation is slower than the other compounds. However, the lignin degradation takes place over $457^{\circ} \mathrm{C}$. This result is fairly similar to that obtained by Kifani-Sahban [11].

Fig. 7 illustrates the radial evolution of the chemical species diffusivity respectively before and after treatment. Accordingly, the thermal treatment helps reduce significantly the diffusion coefficient.

Fig. 7 shows that the model is able to simulate satisfactorily the experimental results of the desorption kinetics for different levels of processing temperatures. The change of the chemical structure which causes an increase in tortuosity of the corck at each level with the presence of the lips at the pore ducts, this structure enhances the mass transfer barrier increasingly with the increase of the treatment temperature level.

In addition to the very satisfactory result related to the decrease of the mass transfer coefficient, we obtain a bio-protection of the crock against insects and a rot. This is due to the degradation of hemicellulose, cellulose and lignin.

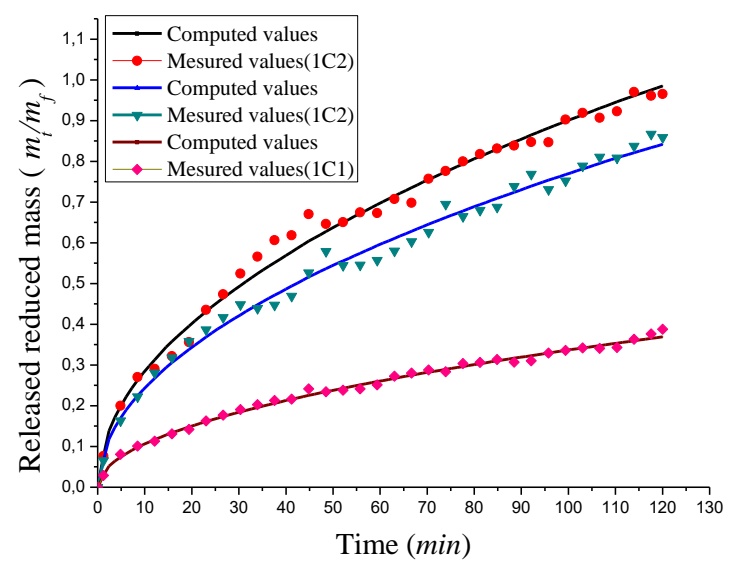

Fig. 7 Variation of the reduced mass released versus time for various treatment temperature

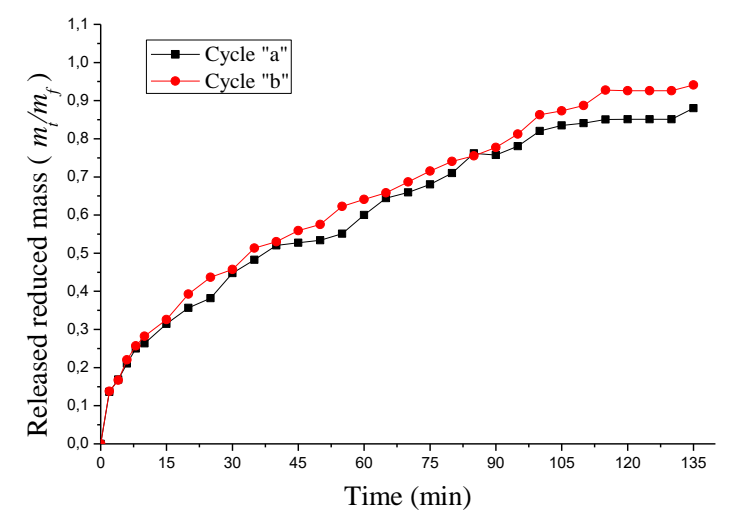

Fig. 8 Variation of the reduced mass released versus time for different cycle
Fig. 8 shows the variation of the released reduced masse versus time for different cycle. It is clear from this figure that this evolution is owing to the decrease of the mass diffusion coefficient. This behavior is may be due to the precipitation of degradation, which leads to the increase of the tortuosity $(\Gamma)$ and shrinkage of the porosity $(\rho)$ of the cork.

The method cited by Champoux et al [12] is used to calculate the tortuosity. Whereas the porosity can be measured by the electrical method [13].

The tortuosity increase can be justified by its direct relationship with the diffusion coefficient given by [14] in our case, Eq. 7:

$$
\Gamma=\sqrt{\frac{5,4033 \times 10^{-8} \times T}{D_{i a p}} \rho} .
$$

Table 3 shows that the apparent diffusion coefficient in the radial direction $\left(D_{R}\right)$ increases with decreasing temperature treatment.

Table 3

Diffusion coefficient under the effect of the treatment temperature (chip 2C)

\begin{tabular}{|c|c|c|c|c|}
\hline Level of treatment, ${ }^{\circ} \mathrm{C}$ & 115 & 370 & 457 & 500 \\
\hline$D_{R} \times 10^{-12}, \mathrm{~m}^{2} \mathrm{~s}^{-1}$ & 6.25 & 4.13 & $9.1410^{-1}$ & $8.0510^{-1}$ \\
\hline
\end{tabular}

\section{Conclusions}

The aim of this study was to improve the mechanical characteristics of cork by using thermal process which causes the degradation of chemicals respectively hemicellulose, cellulose and lignin. In fact, the experimental study has shown that the degradation of hemicelleluses was obtained at the range of temperature between $230<T<370^{\circ} \mathrm{C} D_{R}^{\left(370^{\circ} \mathrm{C}\right)}=4,13 \times 10^{-12} \mathrm{~ms}^{-1}$. The degradation of cellulose is obtained between $370<T<457^{\circ} \mathrm{C}$ $D_{R}^{\left(457^{\circ} \mathrm{C}\right)}=9,14 \times 10^{-13} \mathrm{~ms}^{-1}$, whereas the lignin degradation was significant at the temperature upper than $470^{\circ} \mathrm{C}$. Furthermore, the developed model allows on one hand to simulate the process level on the kinetic of $\mathrm{NaCl}$ desorption and on other hand to evaluate the molecular diffusion coefficient in isothermal conditions. The results obtained show clearly that the thermal process lead to decrease the molecular diffusion coefficient up to 8 times when the cork is treated at $500^{\circ} \mathrm{C} D_{R}^{\left(500^{\circ} \mathrm{C}\right)}=8,05 \times 10^{-13} \mathrm{~ms}^{-1}$. This isolation improvement is due to mechanical change with pore narrowed with an increase in tortuosity.

\section{Acknowledgements}

The authors would like to thank Pr. B. Bezzaai from Boumerdes University for authorization to access to his laboratory to complete experimental measures. 


\section{Reference}

1. Nguila, I.G.; Pétrissans, M.; Lambert, J.L.; Erhardt, J.J.; Gérardin, P. 2006. XPS Characterization of wood chemical composition after heat treatment, Surface \& Interface Analysis 38(10): 1336-1342. http://dx.doi.org/ 10.1002/sia.2455.

2. Nguila, I.G.; Pétrissans, M.; Gérardin, P. 2007. Chemical reactivity of Heat treated Wood, Wood Science Technology 41(2): 157-168. http://dx.doi.org/ 10.1007/s00226-006-0092-7.

3. Esteves, B.; Graça, J.; Pereira, H. 2008. Extractive composition and summative chemical analysis of thermally treated eucalypt wood, Holzforshung 62(1): 344351. http://dx.doi.org/10.1515/HF.2008.057.

4. Gil, L. 2009. Cork composites: a review, Materials 2: 776-789. http://dx.doi.org/10.3390/ma2030776.

5. Chérif, H.-A.; Chaala, A.; Rodrigue, D.; Larachi, F. 2014. Traitement solvothermique superficiel de la biomasse lignocellulosique dans les liquides ioniqueshygroscopicité, morphologie et propriétés mécaniques, Canadian Journal of Chemical Engineering 92(11): 1839-1858. http://dx.doi.org/10.1002/cjce.22095.

6. Mir, A.; Bezzazi, B.; Zitoune, R.; Collombet, F. 2012. Study of mechanical and hygrothermal properties of agglomerated cork, Mechanika 18(1): 40-45. http://dx.doi.org/10.5755/j01.mech.18.1.1278.

7. Agblevor, F.; Besler, S. 1996. Inorganic compounds in biomass feedstocks .1. Effect on the quality of fast pyrolysis oils, Energy \& Fuels 10(2): 293-298. http://dx.doi.org/10.1021/ef950202u.

8. Anderson, D.A.; Freeman, E.S. 2003. The kinetics of the thermal degradation of polystyrene and polyethylene, J. Polymer Science 54(159): 253-260. http://dx.doi.org/10.1002/pol.1961.1205415920.

9. Guimarães, J.L.; Frollini; E.C.G.; da Silva, F.; Wypych, K.G. 2009. Characterization of banana, sugarcane bagasse and sponge gourd fibers of Brazil, Industrial Crops and Products 30(3): 407-415. http://dx.doi.org/10.1016/j.indcrop.2009.07.013.

10. Liu, J.Y.; Simpson, W.T. 1997. Solutions of diffusion equation with constant diffusion and surface emission coefficients, Drying Technology an International Journal 15(10): 64-74. http://dx.doi.org/10.1080/07373939708917370.

11. Kifani-Sahban, F.; Belkbir, L.; Zoulalian, A. 1996.
Study of the slow pyrolysis of Moroccan eucalyptus by thermal analysis, Thermochimica Acta 284(2): 341349.

http://dx.doi.org/10.1016/0040-6031(96)02847-X.

12. Champoux, Y.; Stinson, M.R.; and Daigle, G.A. 1990. Air-based system for the measurement of porosity, J.Acoust.Soc.Am 89: 910-916. http://dx.doi.org/10.1121/1.1894653.

13. Henry, M. 1997. Mesures des paramètres caractérisant un milieu poreux. Etude expérimentale du comportement acoustique des mousses aux basses fréquences. $\mathrm{PhD}$ thesis, Université du Maine. http://www.theses.fr/035022973.

14. Moletta, R. 2009. Traitement Des Déchets, Éd. Tec \& Doc: Lavoisier, 686 p.

http://www.worldcat.org/title/traitement-des-dechets/ oclc/495227388\&referer=brief_results.

\section{T.Kermezli, M.Announ, A.Bensmaili}

\section{THE EFFECT OF THE HEAT TREATMENT LEVEL HT ON THE MECHANICAL BEHAVIOR OF CORK}

S u m m a r y

Sustainability in wet biomaterials embedded in the walls of a building for thermal or sound insulation purpose requires heat treatment, which is known as environment-friendly process, to change some chemical elements responsible for the degradation of the biomaterial. The objective of this work is to study the effect of the temperature of heat treatment cycle in HT on the apparent diffusion coefficient in cork, which was selected based on their ecological characteristics. This coefficient was evaluated indirectly from the measure of the crock conductivity in the radial direction combined with modeling at the range of $10^{-13} \mathrm{~m} / \mathrm{s}$. Likewise, the TGA analysis confirmed that the heat treatment at high temperature, which leads to the degradation of hemicellulose, cellulose and lignin, is more efficient due to the drop of the value of the diffusion coefficient.

Keywords: diffusion coefficient, modeling, cork, Cycle, heat treatment, TGA.

Received May 12, 2015 Accepted January 19, 2016 